\title{
Embedding of a planar rational compactum into a planar continuum with the same rim-type
}

\author{
by
}

\author{
Sophia Zafiridou (Patras)
}

\begin{abstract}
We prove that every planar rational compactum with rim-type $\leq \alpha$, where $\alpha$ is a countable ordinal greater than 0 , can be topologically embedded into a planar rational (locally connected) continuum with rim-type $\leq \alpha$.
\end{abstract}

1. Introduction. All spaces under consideration are separable and metrizable. A space is said to be planar if it is homeomorphic to a subset of the plane. A space is said to be rational if it has a basis consisting of open sets with countable boundaries.

Let $X$ be a space. For every ordinal $\alpha$ the $\alpha$-derivative $X^{(\alpha)}$ of $X$ is defined by induction as follows: $X^{(0)}=X, X^{(\alpha+1)}$ is the set of all limit points of $X^{(\alpha)}$ (in $\left.X^{(\alpha)}\right)$, and $X^{(\alpha)}=\bigcap_{\beta<\alpha} X^{(\beta)}$ for $\alpha$ a limit ordinal ([5], IV, $\S 24)$. If $X^{(\alpha)}=\emptyset$ for some ordinal $\alpha$, then the least such ordinal is called the type of $X$ and is denoted by type $(X)$. Obviously, type $(X)=0$ iff $X=\emptyset$.

It is easy to show that a compactum $X$ is countable iff it has a type. Note that the type of any countable compactum is an isolated countable ordinal and there exists a compactum of type $\alpha$ for any isolated countable ordinal $\alpha([6])$.

The following statements are easily proved and will be used in what follows:

(1.1) If $M$ and $N$ are compacta, type $(M) \leq \alpha$ and type $(N) \leq \alpha$, then type $(M \cup N) \leq \alpha$.

(1.2) If $f$ is a continuous map of a countable compactum $M$ onto $N$, then $\operatorname{type}(N) \leq \operatorname{type}(M)$.

(1.3) If $f$ is a continuous map of a countable compactum $M$ onto $N$ such that $f^{-1}(x)$ is finite for every $x \in N$, then $\operatorname{type}(N)=\operatorname{type}(M)$.

2000 Mathematics Subject Classification: 54C25, 54F50. 
By the rim-type of a rational compactum $X$ is meant the least ordinal $\alpha$ for which there exists a basis of $X$ consisting of open sets whose boundaries are of type $\leq \alpha$. Obviously the rim-type of any rational compactum is a countable ordinal. From now on, $\alpha$ denotes a countable ordinal greater than 0 . Clearly rim-type $(X)=0$ iff $X$ is zero-dimensional.

We quote the following results concerning embeddings of a rational compactum into a rational continuum:

(a) For every countable ordinal $\alpha$ there exists a planar rational (locally connected) continuum with rim-type $\leq \alpha+1$ containing topologically any planar rational compactum with rim-type $\leq \alpha([7])$.

(b) Every rational compactum (not necessarily planar) with rim-type $\leq \alpha$ is contained topologically in a rational continuum with rim-type $\leq \alpha$ ([2]), Theorem 3).

In this paper we prove that every planar rational compactum with rimtype $\leq \alpha$ can be topologically embedded into a planar rational (locally connected) continuum with rim-type $\leq \alpha$.

2. Definitions and notations. Let $E^{2}$ be the plane with a system $O x y$ of orthogonal coordinates. By a disk we mean a subset of $E^{2}$ homeomorphic to $\left\{(x, y) \mid x^{2}+y^{2} \leq 1\right\}$. An arc is a subset $A$ of $E^{2}$ for which there exists a homeomorphism $h$ of the segment $I \equiv[0,1]$ onto $A$. The points $h(0)$ and $h(1)$ are the endpoints of $A$.

By $L_{n}, n=1,2, \ldots$, we denote the set of all ordered $n$-tuples $i_{1} \ldots i_{n}$, where $i_{t}=0$ or $i_{t}=1$, for every $t=1, \ldots, n$. We set $L_{0}=\{\emptyset\}$. For $\bar{i}=i_{1} \ldots i_{n} \in L_{n}, n \geq 1$, we set $\bar{i} 0=i_{1} \ldots i_{n} 0$ and $\bar{i} 1=i_{1} \ldots i_{n} 1$. For $\bar{i}=\emptyset \in L_{0}$ we define $\bar{i} 0=0$ and $\bar{i} 1=1$.

By $I_{\bar{i}}$, where $\bar{i}=i_{1} \ldots i_{n} \in L_{n}, n \geq 1$, we denote the set of all points of $I$ for which the $t$ th digit of the dyadic expansion, $t=1, \ldots, n$, is $i_{t}$. For $\bar{i}=\emptyset \in L_{0}$ we set $I_{\bar{i}}=I_{\emptyset}=I$. We denote by $m(\bar{i})$ the midpoint of $I_{\bar{i}}$.

Let $\mathcal{W}_{n}=\left\{I_{\bar{i}} \times I_{\bar{j}} \mid \bar{i}, \bar{j} \in L_{n}\right\}, n=0,1, \ldots$ Obviously for every $n=$ $0,1, \ldots$ the family $\mathcal{W}_{n}$ is a finite closed covering of $I^{2}$ by disks. We set $\operatorname{Bd}\left(\mathcal{W}_{n}\right)=\bigcup\left\{\operatorname{Bd}(F) \mid F \in \mathcal{W}_{n}\right\}$. Obviously, $\operatorname{Bd}\left(\mathcal{W}_{n}\right) \subseteq \operatorname{Bd}\left(\mathcal{W}_{n+1}\right)$. Note that

$$
\operatorname{Bd}\left(\mathcal{W}_{n+1}\right) \backslash \operatorname{Bd}\left(\mathcal{W}_{n}\right)=\bigcup_{\bar{i}, \bar{j} \in L_{n}}\left(\left(I_{\bar{i}} \times\{m(\bar{j})\}\right) \cup\left(\{m(\bar{j})\} \times I_{\bar{i}}\right)\right)
$$

By $\pi_{1}$ and $\pi_{2}$ we denote the first and the second projection, respectively, of $I^{2}$ onto $I$. We set

$$
\mathcal{V}=\left\{\left(m / 2^{n}, k / 2^{n}\right) \in I^{2} \mid n, m, k=0,1, \ldots\right\} .
$$


Let $C$ be the Cantor ternary set and $C(1)$ be the set of all points of $C$ which are the endpoints of the components of $[0,1] \backslash C$.

For $\bar{i}=i_{1} \ldots i_{n} \in L_{n}, n \geq 1$, we denote by $C_{\bar{i}}$ the set of all points of $C$ for which the $t$ th digit of their ternary expansion, $t=1, \ldots, n$, is 0 if $i_{t}=0$, and 2 if $i_{t}=1$. For $\bar{i}=\emptyset \in L_{0}$ we set $C_{\bar{i}}=C_{\emptyset}=C$. Also we write $a(\bar{i})=\max \left\{x \mid x \in C_{\bar{i} 0}\right\}$ and $b(\bar{i})=\min \left\{x \mid x \in C_{\bar{i} 1}\right\}$ for every $\bar{i} \in \bigcup_{n=0}^{\infty} L_{n}$.

For every $c \in C$ and $n=0,1, \ldots$ we denote by $\bar{i}(c, n)$ the unique $\bar{i} \in L_{n}$ such that $c \in C_{\bar{i}}$. Obviously, $\{c\}=\bigcap_{n=0}^{\infty} C_{\bar{i}(c, n)}$.

3. Lemma. Let $D$ be $a$ disk in the plane, $a, b \in \operatorname{Bd}(D), a \neq b$, and $X \subseteq D \backslash\{a, b\}$ be a rational compactum with rim-type $(X) \leq \alpha$. Then there exists an arc $A \subseteq D$ with endpoints $a, b$ such that type $(A \cap X) \leq \alpha$.

Proof. Let $A_{1}$ and $A_{2}$ be the arcs of $D$ with endpoints $a, b$ such that $A_{1} \cup A_{2}=\operatorname{Bd}(D)$. It is clear that $X \cap A_{1}$ and $X \cap A_{2}$ are closed disjoint subsets of $X \cap D$. Since rim-type $(X \cap D) \leq \alpha$, there exists a closed subset $F$ of $X \cap D$ such that type $(F) \leq \alpha$ and $F$ separates the sets $X \cap A_{1}$ and $X \cap A_{2}$ in $X \cap D$ ([3], Theorem 6).

The rest of the proof which provides an $\operatorname{arc} A \subseteq D$ with endpoints $a, b$ such that $A \cap X \subseteq F$ is the same as the corresponding part of the proof of Lemma 5 in [1].

4. Lemma. For every planar rational compactum $X$ with rim-type $(X)$ $\leq \alpha$ there exists a homeomorphism $h$ of $X$ onto a subset of $I^{2}$ such that:

(1h) $h(X) \subseteq \operatorname{Int}\left(I^{2}\right)$,

(2h) $h(X) \cap \mathcal{V}=\emptyset$,

(3h) type $\left(h(X) \cap \operatorname{Bd}\left(\mathcal{W}_{n}\right)\right) \leq \alpha$ for every $n=1,2, \ldots$

Proof. Let $X$ be a planar rational compactum with $\operatorname{rim}$-type $(X) \leq \alpha$. We set

$$
\begin{aligned}
& Q_{\Delta}=\left\{m / 2^{n} \in I \backslash\{0,1\} \mid m, n=0,1, \ldots\right\}, \\
& Q_{T}=\left\{m / 3^{n} \in I \mid m, n=0,1, \ldots\right\} .
\end{aligned}
$$

Observe that $x \in \operatorname{Int}\left(I^{2}\right) \cap \bigcup_{n=0}^{\infty} \operatorname{Bd}\left(\mathcal{W}_{n}\right)$ iff either $\pi_{1}(x) \in Q_{\Delta}$ or $\pi_{2}(x) \in$ $Q_{\Delta}$, and $x \in \operatorname{Int}\left(I^{2}\right) \cap \mathcal{V}$ iff $\pi_{1}(x), \pi_{2}(x) \in Q_{\Delta}$.

Let $Y=I^{2} \backslash\left(\left(\left(I \backslash Q_{T}\right) \times Q_{\Delta}\right) \cup\left(Q_{\Delta} \times\left(I \backslash Q_{T}\right)\right)\right)$.

It is proved in [1] that $Y$ is a rational containing space for the family of all planar rational compacta. Since $X$ is a planar rational compactum, there exists a homeomorphism $h$ of $X$ onto a subspace of $Y$. Moreover, in [1] the above homeomorphism is constructed in such a manner that condition (1h) is satisfied. Since $Y \cap \operatorname{Int}\left(I^{2}\right) \cap \mathcal{V}=\emptyset$, condition (2h) holds.

Since $Y \cap \operatorname{Bd}\left(\mathcal{W}_{n}\right)$ is countable for every $n=1,2, \ldots$, it follows that $h(X) \cap \operatorname{Bd}\left(\mathcal{W}_{n}\right)$ is countable for every $n=1,2, \ldots$ 
It remains to prove that $h$ satisfies condition (3h). To do that, we slightly modify the construction of $h$ in [1] applying Lemma 3 of the present paper instead of Lemma 5 of [1].

5. Theorem. Every planar rational compactum $X$ with rim-type $(X)$ $\leq \alpha$ can be homeomorphically embedded into a planar rational locally connected continuum $D$ with rim-type $(D) \leq \alpha$.

Proof. Let $X$ be a planar rational compactum with rim-type $(X) \leq \alpha$. By Lemma 4 we can assume that:

(i) $X \subseteq \operatorname{Int}\left(I^{2}\right)$,

(ii) $X \cap \mathcal{V}=\emptyset$,

(iii) type $\left(X \cap \operatorname{Bd}\left(\mathcal{W}_{n}\right)\right) \leq \alpha$ for every $n=1,2, \ldots$

We define a map $f$ of $C^{2}$ onto $I^{2}$ as follows: if $c=\left(c_{1}, c_{2}\right) \in C^{2}$ and $\{c\}=\bigcap_{n=0}^{\infty}\left(C_{\bar{i}\left(c_{1}, n\right)} \times C_{\bar{i}\left(c_{2}, n\right)}\right)$ then $f(c)=\bigcap_{n=0}^{\infty}\left(I_{\bar{i}\left(c_{1}, n\right)} \times I_{\bar{i}\left(c_{2}, n\right)}\right)$. It is easily seen that $f$ has the following properties:

(1f) $f\left(C_{\bar{i}} \times C_{\bar{j}}\right)=I_{\bar{i}} \times I_{\bar{j}}$ for all $\bar{i}, \bar{j} \in L_{n}, n=0,1, \ldots$,

(2f) $f^{-1}(x)$ is a singleton iff neither $\pi_{1}(x)$ nor $\pi_{2}(x)$ is in $Q_{\Delta}$,

(3f) $f^{-1}(x)$ consists of two points iff exactly one of $\pi_{1}(x)$ and $\pi_{2}(x)$ is in $Q_{\Delta}$,

(4f) $f^{-1}(x)$ consists of four points iff $\pi_{1}(x), \pi_{2}(x) \in Q_{\Delta}$,

(5f) $f$ is a continuous map.

Let $x \in X$. Since $X \subseteq \operatorname{Int}\left(I^{2}\right) \backslash \mathcal{V}$, it follows that either $\pi_{1}(x) \notin Q_{\Delta}$ or $\pi_{2}(x) \notin Q_{\Delta}$. From (2f) and (3f) we conclude that $f^{-1}(x)$ consists of at most two points. In particular, $f^{-1}(x)$ is a singleton iff $x \notin \bigcup_{n=0}^{\infty} \operatorname{Bd}\left(\mathcal{W}_{n}\right)$, and $f^{-1}(x)$ is a two-element subset of $C^{2}$ iff $x \in \bigcup_{n=0}^{\infty} \operatorname{Bd}\left(\mathcal{W}_{n}\right)$.

It is easy to verify that

$$
\operatorname{Int}\left(I^{2}\right) \cap \bigcup_{n=0}^{\infty} \operatorname{Bd}\left(\mathcal{W}_{n}\right)=\bigcup_{n=0}^{\infty} \bigcup_{\bar{i}, \bar{j} \in L_{n}}\left(\left(I_{\bar{i}} \times\{m(\bar{j})\}\right) \cup\left(\{m(\bar{j})\} \times I_{\bar{i}}\right)\right) .
$$

Thus $X \cap \bigcup_{n=0}^{\infty} \operatorname{Bd}\left(\mathcal{W}_{n}\right)$ is the union of the sets of the form $X \cap\left(I_{\bar{i}} \times\{m(\bar{j})\}\right)$ and $X \cap\left(\{m(\bar{j})\} \times I_{\bar{i}}\right)$, where $\bar{i}, \bar{j} \in L_{n}, n=0,1, \ldots$

Let $\bar{i}, \bar{j} \in L_{n}, n=0,1, \ldots$ From the definition of $f$ it follows that if $x \in$ $\left(I_{\bar{i}} \times\{m(\bar{j})\}\right) \backslash \mathcal{V}$, then $f^{-1}(x)=\left\{c_{1}, c_{2}\right\}$, where $\pi_{1}\left(c_{1}\right)=\pi_{1}\left(c_{2}\right) \in C_{\bar{i}} \backslash C(1)$ and $\left\{\pi_{2}\left(c_{1}\right), \pi_{2}\left(c_{2}\right)\right\}=\{a(\bar{j}), b(\bar{j})\}$.

Therefore $f^{-1}\left(\left(I_{\bar{i}} \times\{m(\bar{j})\}\right) \backslash \mathcal{V}\right)=\left(C_{\bar{i}} \backslash C(1)\right) \times\{a(\bar{j}), b(\bar{j})\}$. Similarly, $f^{-1}\left(\left(\{m(\bar{j})\} \times I_{\bar{i}}\right) \backslash \mathcal{V}\right)=\{a(\bar{j}), b(\bar{j})\} \times\left(C_{\bar{i}} \backslash C(1)\right)$.

Since $X \cap\left(I_{\bar{i}} \times\{m(\bar{j})\}\right)$ and $X \cap\left(\{m(\bar{j})\} \times I_{\bar{i}}\right)$ are subsets of $\mathrm{Bd}\left(\mathcal{W}_{n+1}\right)$, from the property (iii) of $X$ it follows that type $\left(X \cap\left(I_{\bar{i}} \times\{m(\bar{j})\}\right)\right) \leq \alpha$ and type $\left(X \cap\left(\{m(\bar{j})\} \times I_{\bar{i}}\right)\right) \leq \alpha$. 
Let $\bar{i} \in L_{n}, n=0,1, \ldots$ We set

$$
P_{\bar{i}}^{X}=\bigcup_{\bar{j} \in L_{n}}\left(\pi_{1}\left(f^{-1}\left(X \cap\left(I_{\bar{i}} \times\{m(\bar{j})\}\right)\right)\right) \cup \pi_{2}\left(f^{-1}\left(X \cap\left(\{m(\bar{j})\} \times I_{\bar{i}}\right)\right)\right)\right),
$$

where $\pi_{1}$ (resp. $\pi_{2}$ ) is the projection of $C^{2}$ onto the first (resp. second) coordinate.

Since $X \cap \mathcal{V}=\emptyset$, we have $P_{\bar{i}}^{X} \subseteq C_{\bar{i}} \backslash C(1)$. From (1.1)-(1.3) it follows that $\operatorname{type}\left(P_{\bar{i}}^{X}\right) \leq \alpha$. Since $\alpha>0$, there exists a compact subset $P_{i}$ of $C_{\bar{i}} \backslash C(1)$ such that

(i) $P_{\bar{i}}^{X} \subseteq P_{\bar{i}}$,

(ii) type $\left(P_{\bar{i}}\right)=\operatorname{type}\left(P_{\bar{i}}^{X}\right)$ (therefore type $\left.\left(P_{\bar{i}}\right) \leq \alpha\right)$,

(iii) $P_{i} \cap C_{\bar{i} 0} \neq \emptyset$ and $P_{i} \cap C_{\bar{i} 1} \neq \emptyset$.

For every $\bar{i}, \bar{j} \in L_{n}, n=0,1, \ldots$, we define a collection $D(\bar{i}, \bar{j})$ of twoelement subsets of $C^{2}$ as follows: $\left\{c_{1}, c_{2}\right\} \in D(\bar{i}, \bar{j})$ iff either $\pi_{1}\left(c_{1}\right)=$ $\pi_{1}\left(c_{2}\right) \in P_{i}$ and $\left\{\pi_{2}\left(c_{1}\right), \pi_{2}\left(c_{2}\right)\right\}=\{a(\bar{j}), b(\bar{j})\}$ or $\pi_{2}\left(c_{1}\right)=\pi_{2}\left(c_{2}\right) \in P_{\bar{j}}$ and $\left\{\pi_{1}\left(c_{1}\right), \pi_{1}\left(c_{2}\right)\right\}=\{a(\bar{i}), b(\bar{i})\}$.

We set $D(1)=\bigcup_{n=0}^{\infty}\left\{D(\bar{i}, \bar{j}) \mid \bar{i}, \bar{j} \in L_{n}\right\}$.

Let $D$ be the partition of $C^{2}$ consisting of all elements of $D(1)$ and all singletons $\{c\}$, where $c \in C^{2}$ and $c \notin \bigcup\{d \mid d \in D(1)\}$. It has been proved ([4], Lemma 4) that $D$ is an upper semicontinuous partition of $C^{2}$ and that the corresponding quotient space $D$ is a planar locally connected continuum with rim-type $(D) \leq \alpha$.

Let $p: C^{2} \rightarrow D$ be the quotient mapping. Since the set $f\left(p^{-1}(d)\right)$ is a singleton for every $d \in D$, we can define a mapping $g: D \rightarrow I^{2}$ by letting $g(d)=f\left(p^{-1}(d)\right)$ for $d \in D$. The mapping $g$ is continuous since the composition $f=g \circ p$ is continuous and $p$ is a quotient map. It is easy to see that $\left.g\right|_{g^{-1}(X)}: g^{-1}(X) \rightarrow X$ is one-to-one, hence $g^{-1}(X)$ is homeomorphic to $X$. It follows that $X$ embeds in $D$.

The proof of the theorem is complete.

\section{References}

[1] L. E. Feggos, S. D. Iliadis and S. S. Zafiridou, Planar rational compacta, Colloq. Math. 68 (1995), 49-54.

[2] S. D. Iliadis, On rim-type of spaces, in: Topology (Leningrad, 1982), Lecture Notes in Math. 1060, Springer, New York, 1984, 45-54.

[3] S. D. Iliadis and E. D. Tymchatyn, Compactifications with minimum rim-types of rational spaces, Houston J. Math 17 (1991), 311-323.

[4] S. D. Iliadis and S. S. Zafiridou, Planar rational compacta and universality, Fund. Math. 141 (1992), 109-118.

[5] K. Kuratowski, Topology, Vol. I, Pergamon Press, New York, 1966. 
[6] S. Mazurkiewicz et W. Sierpiński, Contribution à la topologie des ensembles dénombrables, Fund. Math. 1 (1920), 17-27.

[7] J. C. Mayer and E. D. Tymchatyn, Containing spaces for planar rational compacta, Dissertationes Math. 300 (1990).

Department of Mathematics

University of Patras

26500 Patras, Greece

E-mail: zafeirid@math.upatras.gr

Received 20 March 2000;

in revised form 25 October 2000 